\title{
CAPACIDADE FUNCIONAL DE IDOSOS RESIDENTES EM UMA INSTITUIÇÃO DE LONGA PERMANÊNCIA
}

\author{
Luiz Humberto Rodrigues SOUZA*, Ione Fogaça SANTANA \& Selma Santana JESUS
}

Universidade do Estado da Bahia, Campus XII. Guanambi, Bahia, Brasil.

*Autor para correspondência: luizhrsouza21@yahoo.com.br

DOI: http://dx.doi.org/10.18571/acbm.144

\section{RESUMO}

O envelhecimento está associado com as mudanças morfofuncionais que influenciam nas alterações da capacidade funcional $(\mathrm{CF})$ das pessoas idosas. O objetivo desse estudo foi avaliar a CF de idosos residentes em uma instituição de longa permanência, bem como verificar a sua associação com algumas características sociodemográficas e alguns aspectos da saúde física. Trata-se de um estudo transversal. A amostra foi constituída por 32 idosos $(q=18 ; \hat{\sigma}=14)$, com idade igual ou superior a 60 anos, residentes em uma Instituição de Longa Permanência para Idosos (ILPI) no município de Guanambi/BA. Os dados sociodemográficos, aspectos da saúde física e a capacidade em desenvolver as atividades básicas e instrumentais da vida diária foram registrados em uma anamnese. Foi utilizado o teste do Qui-Quadrado $\left(\mathrm{X}^{2}\right)$ para se observar as possíveis associações entre as variáveis. Observou-se prevalência do sexo feminino (56,2\%); a idade média dos voluntários foi de 76,43 $\pm 10,73$ anos. O grau de dependência funcional dos idosos não apresentou associação com o sexo, faixa etária, dados sociodemográficos e indicadores de saúde física $(p>0,05)$. A maioria dos voluntários desse estudo foi considerada semidependente para a realização das atividades básicas e instrumentais da vida diária. Portanto, sugere-se que a inserção de intervenções terapêuticas, como o exercício físico, na rotina dos idosos institucionalizados possa minimizar o efeito de alguns fatores deletérios que influenciam na CF. Assim, é possível que a autonomia e independência dessas pessoas sejam preservadas.

Palavras chave: Capacidade Funcional; Envelhecimento; Instituição de Longa Permanência para Idosos.

\begin{abstract}
Aging is associated with morphofunctional changes that influence the changes in the functional capacity (FC) of the elderly. The objective of this study was to evaluate the FC of elderly residents in a home for the aged, as well as verify its association with some sociodemographic characteristics and some aspects of physical health. This is a cross-sectional study. The sample consisted of 32 elderly $(q=18, \widehat{O}=14)$, aged 60 years or older, living in a Home for the Aged (HA) in the city of Guanambi, Bahia. Socio-demographic data, aspects of physical health and the capacity to develop the basic and instrumental activities of daily living were recorded in an anamnesis. The chi-square test $\left(\mathrm{X}^{2}\right)$ was used to observe the possible associations between the variables. Female prevalence was observed $(56.2 \%)$; the mean age of the volunteers was $76.43 \pm 10.73$ years. The degree of functional dependence of the elderly did not show any association with gender, age group, sociodemographic data and physical health indicators $(p>0.05)$. Most of the volunteers in this study were considered semi-dependent for the basic and instrumental activities of daily living. Therefore, it is suggested that the insertion of therapeutic interventions, such as physical exercise, into the routine of the institutionalized elderly can minimize the effect of some deleterious factors that influence the FC. Thus, it is possible that the autonomy and independence of these people are preserved.
\end{abstract}

Keywords: Functional Capacity; Aging; Homes for the Aged. 


\section{Introdução}

O último censo demonstrou que no período de 2000 a 2020 a população idosa brasileira passará de 13,9 para 28,3 milhões pessoas, chegando em 64 milhões em 2050 (IBGE, 2010). O envelhecimento é um processo natural, dinâmico e progressivo, no qual há alterações morfofuncionais que tornam o organismo mais suscetível ao aparecimento de doenças crônicas não transmissíveis (BRADY et al., 2014). Essas patologias, além de compactuar de maneira negativa na qualidade de vida dos idosos, geram consequências funcionais visíveis que culminam em uma maior vulnerabilidade e dependência na velhice (ALVES et al., 2010).

Durante o envelhecimento, há uma perda do equilíbrio e força muscular, mudanças nos sistemas visual, neurológico e imunológico (REBELATTO et al., 2006; KIRKWOOD et al., 2007) que podem prejudicar a velocidade da marcha (BUSCH et al., 2015) e favorecer a ocorrência de quedas (MUIR et al., 2012). O principal desfecho decorrente dessas mudanças é o comprometimento da capacidade funcional (CF) do idoso, que resulta no aumento com o seu cuidado/atenção pela família e com os gastos da saúde pública (BRITO et al., 2013).

A CF é definida como a habilidade física e mental necessária para manter o autocuidado do idoso, o qual influencia na preservação da sua autonomia e independência durante a realização das atividades rotineiras (COSTA et al., 2006; PELEGRIN et al., 2008). De uma maneira mais simples, a CF corresponde a ausência de dificuldade no desempenho de certos gestos e/ou atividades da vida cotidiana (LIMA-COSTA et al., 2003). Ela é influenciada por fatores sociodemográficos, econômicos, culturais e psicossociais (COSTA et al., 2006; PELEGRIN et al., 2008) e pode ser avaliada de acordo com o desempenho do idoso durante a execução das atividades básicas (autocuidado, mobilidade, alimentação, higiene pessoal) e instrumentais (integram o idoso na comunidade; cuidado intradomiciliar) da vida diária (CALDAS, 2003).

Em idosos institucionalizados, esse cenário é mais crítico, pois sua capacidade em realizar algo com os próprios meios é mais limitada (RAPOSO et al., 2017). As instituições de longa permanência para idosos (ILPI) têm a finalidade de cuidar de pessoas idosas que possuem alguma dificuldade para executar as atividades da vida diária (AVD) e/ou estão impossibilitadas de receber os cuidados necessários de seus familiares (CAMARANO, 2008). De acordo com o levantamento de caráter censitário, no Brasil há 3.548 ILPI, as quais dão assistência para 95.200 pessoas idosas; em média, cada ILPI brasileira abriga 30,4 residentes, inferindo, portanto, que as instituições brasileiras são pequenas, pois predominam aquelas que abrigam menos de 20 residentes (CAMARANO et al., 2010). Foi verificado que a redução da mobilidade funcional de idosos institucionalizados interferiu significativamente na realização das atividades de transferência, banho e vestuário, ratificando assim, que a mobilidade é um componente da função física extremamente importante para a execução das AVD e a manutenção da independência (OLIVEIRA et al., 2006). A partir dessa evidência, nota-se que o declínio funcional é um marcador para uma possível manifestação de fragilidade no idoso, e por isso deve ser considerado uma prioridade para a intervenção geriátrica e gerontológica (RAPOSO et al., 2017). Portanto, a avaliação da CF do idoso é importante para determinar o risco de dependência futura e direcionar o melhor tipo de intervenção/monitoração do estado funcional neste grupo (CAMARA et al., 2008), de tal modo que possa contribuir na preservação da sua autonomia (tomada de decisão) e independência (execução), permitindo que o indivíduo cuide de si e de sua vida (BRASIL, 2006).

Sendo assim, o objetivo desse estudo foi avaliar a CF de idosos residentes em uma instituição de longa permanência, bem como verificar a sua associação com algumas características sociodemográficas e alguns aspectos da saúde física. 


\section{Biomedica Brasiliensia}

\section{Materiais e Métodos}

Trata-se de um estudo exploratório, descritivo e inferencial, de corte transversal. A coleta de dados aconteceu na Associação Benemérita de Caridade (Lar dos Velhinhos) localizada na cidade de Guanambi, Bahia. A instituição possui 62 internos $(\widehat{\sigma}=35$ e $q=27)$. Destes, 36 possuem idade igual ou superior a 60 anos. A amostra deste estudo foi selecionada por conveniência, de maneira não probabilística, a qual foi composta por 32 idosos sedentários ( $\widehat{O}=$ 14 e $q=18$ ), pois 2 idosos optaram por não participar e 2 idosos não responderam todos os itens das escalas. Essas pessoas foram informadas sobre os procedimentos da pesquisa e concordaram em participar, voluntariamente, por meio da assinatura do Termo de Consentimento Livre e Esclarecido. Foram adotados os seguintes critérios de inclusão: idosos com idade igual ou superior a 60 anos, de ambos os sexos, ser residente na associação benemérita de caridade. Quanto aos critérios de exclusão, adotou-se: idosos que não responderam todos os itens das escalas e que não aceitaram participar da pesquisa.

Para a avaliação dos idosos foi elaborada uma anamnese que constou dos seguintes itens: $1^{\text {o }}$ - Aspectos sociodemográficos: sexo, idade, religião, cor da pele, estado civil, escolaridade, ocupação; $2^{\circ}$ - Aspectos da saúde física: uso de medicamentos e patologias autorreferidas; $3^{\circ}$ Para avaliar a capacidade em desenvolver as AVD, utilizou-se a Escala de Katz (KATZ et al. 1963), validada para a população brasileira (LINO et al., 2008). O grau de dependência funcional (independente, semidependente e dependente) seguiu as recomendações de Afonso et al. (2013). $4^{\circ}$ - Para avaliar a capacidade em desenvolver as atividades instrumentais da vida diária (AIVD), utilizou-se a Escala de Lawton e Brody (1969). O grau de dependência funcional (independente, semidependente e dependente) seguiu as recomendações de Florianópolis (2011).

Todas as análises foram realizadas com o pacote estatístico IBM SPSS versão 20.0 (SPSS, Inc., Chicago, IL, EUA). Utilizou-se a estatística descritiva com a distribuição de frequência absoluta e relativa para variáveis categóricas e a média com desvio padrão para as variáveis contínuas. Na estatística inferencial realizou-se uma análise bivariada utilizando-se o Teste do Qui-Quadrado $\left(\mathrm{X}^{2}\right)$ de Pearson para se observarem as possíveis associações existentes entre as variáveis independentes e a dependente. O nível de significância adotado foi de $p<0,05$.

Este estudo atendeu aos requisitos propostos pela Resolução no 466/2012 do Conselho Nacional de Saúde (CNS) e foi aprovado pelo Comitê de Ética em Pesquisa com seres humanos do Instituto Avançado de Ensino Superior de Barreiras - IAESB sob o parecer n 405.322/2013.

\section{Resultados}

A amostra incluiu 56,2\% de mulheres e apresentou uma idade média de 76,43 $\pm 10,73$ anos (variando de 60 a 98 anos). Aproximadamente, $82 \%$ dos voluntários pertenciam a religião católica, $100 \%$ eram aposentados, 46,9\% relataram a cor da pele branca e analfabetas e $40,6 \%$ eram solteiras. Em relação ao uso de medicamentos e às patologias autorreferidas, $68,8 \%$ e $75 \%$ da amostra, respectivamente, faziam uso de pelo menos um medicamento continuamente e possuíam pelo menos uma doença crônica não transmissível (Tabela 1).

A classificação "semidependente" para o grau de dependência funcional das AVD foi prevalente $(53,1 \%)$ nesta amostra estudada. Todavia, a análise inferencial do teste qui-quadrado sinalizou que não houve evidências de associação entre o grau de dependência funcional dos idosos com o sexo $\left(X^{2}=4,59 ; p=0,10\right)$, faixa etária $\left(X^{2}=3,63 ; p=0,46\right)$, dados sociodemográficos $(p$ $>0,05)$ e indicadores de saúde física ( $p>0,05$; Tabela 1). Outrossim, houve prevalência da classificação "semidependente" $(65,6 \%)$ para o grau de dependência funcional das AIVD. Verificou-se que não houve associação entre o grau de dependência funcional dos idosos com o sexo $\left(\mathrm{X}^{2}=5,78 ; p=0,06\right)$, faixa etária $\left(\mathrm{X}^{2}=3,14 ; p=0,54\right)$, dados sociodemográficos $(p>0,05)$ e indicadores de saúde física $(p>0,05$; Tabela 2$)$. 
Tabela 1: Grau de dependência (AVD) vs. dados sociodemográficos e indicadores da saúde física.

\begin{tabular}{|c|c|c|c|c|c|c|}
\hline \multirow{2}{*}{ Variáveis } & \multirow{2}{*}{ Classes } & \multicolumn{3}{|c|}{ AVD } & \multirow{2}{*}{ TOTAL } & \multirow{2}{*}{$X^{2}(p)$} \\
\hline & & I & SD & D & & \\
\hline \multirow{2}{*}{ Sexo } & Masculino & $9(28,1 \%)$ & $5(15,6 \%)$ & $0(0 \%)$ & $14(43,8 \%)$ & \multirow{2}{*}{$4,6(0,10)$} \\
\hline & Feminino & $5(15,6 \%)$ & $12(37,5 \%)$ & $1(3,1 \%)$ & $18(56,2 \%)$ & \\
\hline \multirow{3}{*}{ Faixa Etária } & 60 a 69 anos & $5(15,6 \%)$ & $4(12,5 \%)$ & $0(0 \%)$ & $9(28,1 \%)$ & \multirow{3}{*}{$3,6(0,46)$} \\
\hline & 70 a 79 anos & $4(12,5 \%)$ & $4(12,5 \%)$ & $1(3,1 \%)$ & $9(28,1 \%)$ & \\
\hline & $\geq 80$ anos & $5(15,6 \%)$ & $9(28,1 \%)$ & $0(0 \%)$ & $14(43,8 \%)$ & \\
\hline \multirow{2}{*}{ Religião } & Católica & $10(31,2 \%)$ & $15(46,9 \%)$ & $1(3,1 \%)$ & $26(81,2 \%)$ & \multirow{2}{*}{$1,7(0,44)$} \\
\hline & Evangélica & $4(12,5 \%)$ & $2(6,2 \%)$ & $0(0 \%)$ & $6(18,8 \%)$ & \\
\hline \multirow{3}{*}{ Cor da Pele } & Negra & $2(6,2 \%)$ & $1(3,1 \%)$ & $0(0 \%)$ & $3(9,4 \%)$ & \multirow{3}{*}{$4,1(0,40)$} \\
\hline & Branca & $4(12,5 \%)$ & $10(31,2 \%)$ & $1(3,1 \%)$ & $15(46,9 \%)$ & \\
\hline & Parda & $8(25 \%)$ & $6(18,8 \%)$ & $0(0 \%)$ & $14(43,8 \%)$ & \\
\hline \multirow{4}{*}{ Estado Civil } & Solteiro & $5(15,6 \%)$ & $7(21,9 \%)$ & $1(3,1 \%)$ & $13(40,6 \%)$ & \multirow{4}{*}{$3,3(0,78)$} \\
\hline & Divorciado & $3(9,4 \%)$ & $4(12,5 \%)$ & $0(0 \%)$ & $7(21,9 \%)$ & \\
\hline & Viúvo & $5(15,6 \%)$ & $3(9,4 \%)$ & $0(0 \%)$ & $8(25 \%)$ & \\
\hline & Casado & $1(3,1 \%)$ & $3(9,4 \%)$ & $0(0 \%)$ & $4(12,5 \%)$ & \\
\hline \multirow{3}{*}{ Escolaridade } & Analfabeto & $5(15,6 \%)$ & $9(28,1 \%)$ & $1(3,1 \%)$ & $15(46,9 \%)$ & \multirow{3}{*}{$9,6(0,05)$} \\
\hline & Lê/Escreve & $2(6,2 \%)$ & $7(21,9 \%)$ & $0(0 \%)$ & $9(28,1 \%)$ & \\
\hline & $1^{\circ} \mathrm{GI}$ & $7(21,9 \%)$ & $1(3,1 \%)$ & $0(0 \%)$ & $8(25 \%)$ & \\
\hline \multirow{2}{*}{ Medicamento } & Sim & $10(31,2 \%)$ & $11(34,4 \%)$ & $1(3,1 \%)$ & $22(68,8 \%)$ & \multirow{2}{*}{$0,6(0,73)$} \\
\hline & Não & $4(12,5 \%)$ & $6(18,8 \%)$ & $0(0 \%)$ & $10(31,2 \%)$ & \\
\hline \multirow{2}{*}{ Patologia } & Sim & $11(34,4 \%)$ & $12(37,5 \%)$ & $1(3,1 \%)$ & $24(75 \%)$ & \multirow{2}{*}{$0,6(0,74)$} \\
\hline & Não & $3(9,4 \%)$ & $5(15,6 \%)$ & $0(0 \%)$ & $8(25 \%)$ & \\
\hline \multicolumn{2}{|c|}{ TOTAL } & $14(43,8 \%)$ & $17(53,1 \%)$ & $1(3,1 \%)$ & $32(100 \%)$ & \\
\hline
\end{tabular}

$\mathrm{AVD}=$ atividades da vida diária; $\mathrm{I}=$ independente; $\mathrm{SD}=$ semidependente $; \mathrm{D}=$ dependente $; 1^{\circ} \mathrm{GI}=$ primeiro grau incompleto.

Tabela 2: Grau de dependência (AIVD) vs. dados sociodemográficos e indicadores da saúde física.

\begin{tabular}{|c|c|c|c|c|c|c|}
\hline \multirow{2}{*}{ Variáveis } & \multirow{2}{*}{ Classes } & \multicolumn{3}{|c|}{ AIVD } & \multirow{2}{*}{ TOTAL } & \multirow{2}{*}{$\mathrm{X}^{2}(p)$} \\
\hline & & I & SD & D & & \\
\hline \multirow{2}{*}{ Sexo } & Masculino & $6(18,8 \%)$ & $8(25 \%)$ & $0(0 \%)$ & $14(43,8 \%)$ & \multirow{2}{*}{$5,8(0,06)$} \\
\hline & Feminino & $2(6,2 \%)$ & $13(40,6 \%)$ & $3(9,4 \%)$ & $18(56,2 \%)$ & \\
\hline \multirow{3}{*}{ Faixa Etária } & 60 a 69 anos & $4(12,5 \%)$ & $4(12,5 \%)$ & $1(3,1 \%)$ & $9(28,1 \%)$ & \multirow{3}{*}{$3,1(0,54)$} \\
\hline & 70 a 79 anos & $2(6,2 \%)$ & $6(18,8 \%)$ & $1(3,1 \%)$ & $9(28,1 \%)$ & \\
\hline & $\geq 80$ anos & $2(6,2 \%)$ & $11(34,4 \%)$ & $1(3,1 \%)$ & $14(43,8 \%)$ & \\
\hline \multirow{2}{*}{ Religião } & Católica & $5(15,6 \%)$ & $18(56,2 \%)$ & $3(9,4 \%)$ & $26(81,2 \%)$ & \multirow{2}{*}{$2,8(0,25)$} \\
\hline & Evangélica & $3(9,4 \%)$ & $3(9,4 \%)$ & $0(0 \%)$ & $6(18,8 \%)$ & \\
\hline \multirow{3}{*}{ Cor da Pele } & Negra & $1(3,1 \%)$ & $1(3,1 \%)$ & $1(3,1 \%)$ & $3(9,4 \%)$ & \multirow{3}{*}{$4,3(0,37)$} \\
\hline & Branca & $3(9,4 \%)$ & $10(31,2 \%)$ & $2(6,2 \%)$ & $15(46,9 \%)$ & \\
\hline & Parda & $4(12,5 \%)$ & $10(31,2 \%)$ & $0(0 \%)$ & $14(43,8 \%)$ & \\
\hline \multirow{4}{*}{ Estado Civil } & Solteiro & $2(6,2 \%)$ & $9(28,1 \%)$ & $2(6,2 \%)$ & $13(40,6 \%)$ & \multirow{4}{*}{$3,2(0,79)$} \\
\hline & Divorciado & $3(9,4 \%)$ & $4(12,5 \%)$ & $0(0 \%)$ & $7(21,9 \%)$ & \\
\hline & Viúvo & $2(6,2 \%)$ & $5(15,6 \%)$ & $1(3,1 \%)$ & $8(25 \%)$ & \\
\hline & Casado & $1(3,1 \%)$ & $3(9,4 \%)$ & $0(0 \%)$ & $4(12,5 \%)$ & \\
\hline \multirow{3}{*}{ Escolaridade } & Analfabeto & $1(3,1 \%)$ & $12(37,5 \%)$ & $2(6,2 \%)$ & $15(46,9 \%)$ & \multirow{3}{*}{$9,0(0,06)$} \\
\hline & Lê/Escreve & $2(6,2 \%)$ & $6(18,8 \%)$ & $1(3,1 \%)$ & $9(28,1 \%)$ & \\
\hline & $1^{\circ} \mathrm{GI}$ & $5(15,6 \%)$ & $3(9,4 \%)$ & $0(0 \%)$ & $8(25 \%)$ & \\
\hline \multirow{2}{*}{ Medicamento } & Sim & $7(21,9 \%)$ & $13(40,6 \%)$ & $2(6,2 \%)$ & $22(68,8 \%)$ & \multirow{2}{*}{$1,8(0,41)$} \\
\hline & Não & $1(3,1 \%)$ & $8(25 \%)$ & $1(3,1 \%)$ & $10(31,2 \%)$ & \\
\hline \multirow{2}{*}{ Patologia } & Sim & $8(25 \%)$ & $14(43,8 \%)$ & $2(6,2 \%)$ & $24(75 \%)$ & \multirow{2}{*}{$3,6(0,17)$} \\
\hline & Não & $0(0 \%)$ & $7(21,9 \%)$ & $1(3,1 \%)$ & $8(25 \%)$ & \\
\hline \multicolumn{2}{|c|}{ TOTAL } & $8(25 \%)$ & $21(65,6 \%)$ & $3(9,4 \%)$ & $32(100 \%)$ & \\
\hline
\end{tabular}

$\mathrm{AIVD}=$ atividades instrumentais da vida diária; $\mathrm{I}=$ independente; $\mathrm{SD}=$ semidependente $; \mathrm{D}=$ dependente; $1^{\circ} \mathrm{GI}=$ primeiro grau incompleto. 


\section{Discussão}

Este estudo avaliou 32 idosos institucionalizados, dos quais 56,2\% foram mulheres. Essa informação converge com os dados apresentados na área do envelhecimento, em que a maioria dos participantes são mulheres idosas (ARAÚJO e CEOLIM, 2007; PELEGRIN et al., 2008; SUDRÉ et al., 2012; MARINHO et al., 2013; BARBOSA et al., 2014). Veras (1994) explicou que as mulheres vivem mais pois são menos expostas a riscos, acidentes de trabalho e de trânsito, homicídios, suicídios, consomem menos tabaco e álcool e fazem uso mais frequente dos serviços de saúde.

A escala de Katz foi desenvolvida devido ao número crescente da população idosa e à prevalência de doenças crônicas neste público, dando ênfase à questão da avaliação funcional (DUARTE et al., 2007). Ela avalia as tarefas necessárias para o cuidado com o corpo ou autopreservação (KATZ et al., 1963). Por esses motivos, optamos em utilizá-la neste estudo. Observou-se que a maioria dos voluntários foi classificada como "semidependente" para o grau de dependência funcional das $\operatorname{AVD}(53,1 \%)$, sendo que as áreas de funcionamento mais comprometidas foram "tomar banho" e "vestir-se". Embora o presente estudo não tenha encontrado associação entre o grau de dependência funcional e o sexo dos voluntários $(p=0,10)$, previamente, foi verificado que mulheres institucionalizadas foram consideradas mais independentes que os homens (ARAÚJO e CEOLIM, 2007; PELEGRIN et al., 2008) e as atividades funcionais mais comprometidas foram tomar banho e vestir-se (PELEGRIN et al., 2008). Todavia, mais recentemente, Marinho et al. (2013) também não encontraram diferença significativa ao comparar o índice de Katz entre os sexos de idosos institucionalizados.

Em nosso estudo, houve uma prevalência de idosos com idade igual ou superior a 80 anos (43,8\%), embora não foi encontrada associação dessa variável com o grau de dependência funcional da AVD. Por outro lado, Araújo e Ceolim (2007) verificaram que o declínio funcional ocorreu, predominantemente, em idosos institucionalizados com 80 anos ou mais de idade. Igualmente, foi constatado um aumento da ocorrência de dependência funcional com o aumento da idade (MARINHO et al., 2013). Em idosos não institucionalizados, assistidos pela Estratégia da Saúde da Família, notou-se que a maioria dos idosos considerados dependentes era do sexo feminino e estava na faixa etária de 70 a 79 anos (SUDRÉ et al., 2012).

Os dados sociodemográficos e os indicadores de saúde física também não se associaram com o grau de dependência funcional dos nossos voluntários. Porém, notamos que o nível de escolaridade apresentou uma tendência de associação $(p=0,05)$. Foi demonstrado que idosos com menor nível de escolaridade possuíam menor conhecimento sobre prevenção de agravos à saúde e tinham menos acesso à rede de saúde por meio de planos privados de atendimento (ALEXANDRE et al., 2009). Verificamos que os betabloqueadores, diuréticos tiazídicos, antagonistas do receptor da angiotensina II, inibidores da enzima conversora da angiotensina foram os medicamentos mais usados pelos idosos, já que a hipertensão arterial foi a patologia prevalente neste grupo. Da mesma forma, Melo e Saintrain (2009) detectaram que a hipertensão foi a patologia mais comum em seu grupo (35,5\%), sendo que $71,0 \%$ estavam fazendo uso de medicamentos para tratamento e controle de suas enfermidades. Essas informações servem como alerta, pois foi constatado que idosos do sexo feminino, idosos com idade avançada (> 80 anos), que viviam sem companheiro (solteiros, separados e viúvos), não possuíam escolaridade formal e apresentavam incapacidade funcional para AVD apresentam maior probabilidade de institucionalização (DEL DUCA et al., 2012).

A escala de Lawton e Brody (1969) é um instrumento de fácil aplicação e interpretação. Ela avalia as AIVD que englobam as tarefas relacionadas com a participação do idoso no contexto social e nas atividades da família. Verificou-se que a maioria dos voluntários se classificou como "semidependente" $(65,6 \%)$ para o grau de dependência funcional a partir das AIVD, sendo que as atividades mais comprometidas foram "lavar/passar a roupa" e "trabalho doméstico/manual". Neste estudo, o sexo, faixa etária, dados sociodemográficos e indicadores da saúde física dos 
voluntários não se associaram com o grau de dependência das AIVD ( $p>0,05)$, embora uma tendência de associação com o sexo e escolaridade foi percebida $(\mathrm{p}=0,06)$. Por outro lado, Oliveira e Mattos (2012) verificaram que a prevalência de dependência para AIVD $(88,7 \%)$ foi associada ao sexo feminino, ausência de contato familiar e déficit cognitivo em idosos institucionalizados, e essa prevalência nos idosos institucionalizados foi 3 vezes maior do que a observada em idosos da comunidade, embora os fatores de risco associados à dependência sejam similares nos dois grupos. Não obstante, Barbosa et al. (2014) mostraram que, em idosos não institucionalizados, a dependência funcional nas AIVD foi associada à faixa etária $\geq 75$ anos, ao sexo feminino e a presença de doença cardíaca. Esse estudo ainda mostrou que as maiores dependências dos idosos foram ir a lugares mais distantes sozinhos, lavar e passar roupa e usar o telefone.

Em nosso estudo, foi constatado que houve prevalência na dependência das AIVD (dependente, 9,4\%; semidependente, 65,6\%; independente, 25\%) em relação as AVD (dependente, $3,1 \%$; semidependente, $53,1 \%$; independente, $43,8 \%$ ) dos idosos, o que está de acordo com outras pesquisas (MILLÁN-CALENTI et al., 2010; BARBOSA et al., 2014). As perdas funcionais ocorrem das AIVD para as AVD, pois as AIVD exigem maior integridade física e cognitiva em relação às AVD (MILLÁN-CALENTI et al., 2010). Porém, isso é preocupante, pois as AIVD incluem problemas mais complexos da vida cotidiana e são indicadores de funções sociais como gerir o orçamento doméstico, utilizar telefone, sair sozinho e fazer compras (MATSUDO, 2010). Costa et al. (2006) comungam dessa ideia, pois afirmam que a dependência funcional do idoso pode levar à perda de autonomia tornando-se necessário a ajuda de outras pessoas para gerir seus bens financeiros, fazer suas compras e realizar os afazeres domésticos. Ademais, a incapacidade do idoso para realizar as AVD e AIVD, além de prejudicar a vida social do idoso, potencialmente implica em transtornos para ele e sua família.

Por outro lado, foi verificado que idosos praticantes de musculação e atividades rítmicas/recreativas apresentaram melhores níveis de autonomia para o desempenho de suas atividades cotidianas, enquanto os idosos sedentários apresentavam maior dificuldade e até mesmo dependência (BORGES e MOREIRA, 2009). Essa evidência sugere que o exercício físico é uma alternativa que pode minimizar as alterações indesejáveis na CF dos idosos. Além disso, observouse uma tendência inversa entre a ocorrência de institucionalização do idoso e o nível de atividade física, em que sujeitos pouco ativos e inativos apresentaram maiores probabilidades de institucionalização (DEL DUCA et al., 2012). Portanto, percebe-se que o perfil para um envelhecimento bem-sucedido está associado a uma CF funcional mais aprimorada. Sendo assim, espera-se que um idoso mais autônomo e com melhor desempenho na realização das AVD e AIVD distancie-se da dependência funcional em grau elevado.

\section{Conclusão}

A maioria dos voluntários desse estudo foi considerada semidependente para a realização das AVD e AIVD. Sendo assim, algumas habilidades físicas, funcionais e cognitivas necessárias para a sua autonomia e independência funcional, provavelmente, já foram acometidas. Portanto, sugere-se que a inserção de intervenções terapêuticas, como o exercício físico, na rotina desses idosos institucionalizados possa minimizar o efeito de alguns fatores deletérios que influenciam na CF. Desse modo, é possível que as atividades do autocuidado e as tarefas domésticas do dia a dia dessas pessoas sejam preservadas.

Não houve associação entre o grau de dependência funcional desses voluntários com sexo, faixa etária, dados sociodemográficos e indicadores de saúde física, embora, em termos percentuais, tanto para as AVD quanto para as AIVD, o sexo feminino, faixa etária acima de 80 anos, estado civil solteiro, nível de escolaridade analfabeto, usuários de medicamentos e presença de patologia tenham sido prevalentes na classificação funcional semidependente. 
Por outro lado, algumas limitações devem ser destacadas: (I) embora os instrumentos utilizados nesse trabalho sejam validados e reportados de maneira recorrente na literatura, é preciso registrar que as informações foram sinalizadas pelos próprios voluntários. Para garantir a segurança dessas informações, os pesquisadores confirmaram a veracidade com os cuidadores da ILPI; (II) durante a realização da pesquisa, a ILPI possuía 62 internos, dos quais 26 tinham idade entre 48 e 59 anos (não foram incluídos no estudo). Embora a associação benemérita de caridade receba o nome de "Lar dos Velhinhos", nem todos os moradores enquadram-se na classificação idosa (60 anos ou mais). Isso acontece por que este espaço é o único lugar da cidade que abriga pessoas em situações de risco. Portanto, apenas 4 idosos da ILPI não participaram do estudo, perfazendo uma amostra de 32 idosos. Nós avaliamos esse tamanho amostral pequeno para uma análise inferencial robusta. Sendo assim, sugerimos que as generalizações dos resultados desse estudo sejam feitas cautelosamente; e (III) o delineamento de corte transversal desse estudo nos impossibilitou verificar os fatores de risco, ao longo do tempo, para a dependência funcional entre os idosos institucionalizados. Isso é importante, pois pode auxiliar no planejamento de ações que promovam a saúde, qualidade de vida e bem-estar desta população. Além disso, sugerimos uma intervenção com exercício físico para verificar o seu efeito sobre o desempenho das ADV e AIVD, que são marcadores da CF.

\section{Referências}

AFONSO, M. S.; SILVEIRA, K. S.; SOARES, M. P.; LOPES, J. F.; AZEVEDO, P.; BRITO, L. C. N. Déficits funcionais de idosos correlacionados a cada década de vidas. Rev Inspirar Mov Saude, v. 5, n. 2, p. 1-6, 2013. Disponível em: < https://www.inspirar.com.br/novosite/wpcontent/uploads/2014/10/deficitis-funcionais-artigo-279.pdf >. Acessado em 20 de julho de 2017.

ALEXANDRE, T. S.; CORDEIRO, R. C.; RAMOS, L. R. Factors associated to quality of life in active elderly. Rev Saude Publica, v. 43, n. 4, p. 613-621, 2009.

ALVES, L. C.; LEITE, I. C.; MACHADO, C. J. Factors associated with functional disability of elderly in Brazil: a multilevel analysis. Rev Saude Publica, v. 44, n. 3, p. 468-478, 2010.

ARAÚJO, M. O. P. H.; CEOLIM, M. F. Assessment of the level of independence of elderly residents in long-term care institutions. Rev Esc Enferm USP, v. 41, n. 3, p. 378-385, 2007.

BARBOSA, B. R.; ALMEIDA, J. M.; BARBOSA, M. R.; BARBOSA, L. A. R. R. Evaluation of the functional capacity of the elderly and factors associated with disability. Cien Saude Colet, v. 19, n. 8, p. 3317-3325, 2014.

BORGES, M. R. D.; MOREIRA, A. K. Influências da prática de atividades físicas na terceira idade: estudo comparativo dos níveis de autonomia para o desempenho nas AVDs e AIVDs entre idosos ativos fisicamente e idosos sedentários. Motriz, v. 15, n. 3, p. 562-573, 2009.

BRADY, A. O.; STRAIGHT, C. R.; EVANS, E. M. Body composition, muscle capacity, and physical function in older adults: an integrated conceptual model. J Aging Phys Act, v. 22, n. 3, p. 441-452, 2014.

BRASIL. Ministério da Saúde. Política Nacional de Saúde da Pessoa Idosa. Portaria MS/GM no 2.528 de 19 de outubro de 2006 . Aprova a política nacional de saúde da pessoa idosa. Brasília: Ministério da $2006 . \quad$ Saúde, Disponível em: 
http://bvsms.saude.gov.br/bvs/saudelegis/gm/2006/prt2528_19_10_2006.html>. Acesso em 05 maio 2017.

BRITO, M. C. C.; FREITAS, C. A. S. L.; MESQUITA, K. O.; LIMA, G. K. Envelhecimento populacional e os desafios para a saúde pública: análise da produção científica. Rev Kairos, v. 16, n. 2, p. 161-178, 2013.

BUSCH, T. A.; DUARTE, Y. A.; NUNES, D. P.; LEBRÃO, M. L.; NASLAVSKY, M. S.; RODRIGUES, A. S.; AMARO, E. Factors associated with lower gait speed among the elderly living in a developing country: a cross-sectional population-based study. BMC Geriatr, v. 15, p. 35-43, 2015.

CALDAS, C. P. Aging with dependence: family needs and responsibilities. Cad Saude Publica, v. 19, n. 3, p. 773-781, 2003.

CAMARA, F. M.; GEREZ, A. G.; MIRANDA, M. L. J.; VELARDI, M. Elderly functional capacity: types of assessment and trends. Acta Fisiatrica, v. 15, n. 4, p. 249-256, 2008.

CAMARANO, A. A. Características das instituições de longa permanência para idosos: região centro-oeste. Brasília: IPEA, 2008.

CAMARANO, A. A.; KANSO, S.; MELLO, J. L.; CARVALHO, D. F. As instituições de longa permanência para idosos no Brasil. In: CAMARANO, A. A. (Org.), Cuidados de longa duração para a população idosa: um novo risco social a ser assumido? Rio de Janeiro: IPEA, 2010.

COSTA, E. C.; NAKATANI, A. I. K.; BACHION, M. M. Elder's community capacity to develop daily life activities and daily instrumental life activities. Acta Paul Enferm, v. 19, n. 1, p. 43-48, 2006.

DEL DUCA, G. F.; SILVA, S. G.; THUMÉ, E.; SANTOS, I. S.; HALLAL, P. C. Predictive factors for institutionalization of the elderly: a case-control study. Rev Saude Publica, v. 46, n. 1, p. 147$153,2012$.

DUARTE, Y. A.; ANDRADE, O. C. L.; LEBRÃO, M. L. Katz index on elderly functionality evaluation. Rev Esc Enferm USP, v. 41, n. 2, p. 317-325, 2007.

FLORIANÓPOLIS. Secretaria Municipal de Saúde. Diretoria da Atenção Primária à Saúde. Protocolo de atenção à saúde do idoso. Tubarão: Copiart, 2011. Disponível em: <http://portal.pmf.sc.gov.br/arquivos/arquivos/pdf/14_05_2012_8.47.51.ea16b1f5291407e4d39d 30837dfc2809.pdf $>$. Acesso em 15 junho 2017.

INSTITUTO BRASILEIRO DE GEOGRAFIA E ESTATÍSTICA. Indicadores sociais municipais: uma análise dos resultados do universo do censo demográfico 2010. Estudos e pesquisas: informação demográfica e socioeconômica. Rio de Janeiro: Ministério do Planejamento, Orçamento e Gestão, 2011. Disponível em: <http://biblioteca.ibge.gov.br/visualizacao/livros/liv54598.pdf>. Acesso em 11 abril 2017. 


\section{Biomedica Brasiliensia}

ISSN: 2236-0867

KATZ, S.; FORD, A. B.; MOSKOWITZ, R. W.; JACKSON, B. A.; JAFFE, M. W. Studies of illness in the aged. The index of ADL: a standardized measure of biological and psychosocial function. Jama, v. 185, n. 12, p. 914-919, 1963.

KIRKWOOD, R. N.; GOMES, H. A.; SAMPAIO, R. F.; CULHAM, E.; COSTIGAN, P. Biomechanical analysis of hip and knee joints during gait in elderly subjects. Acta Ortop Bras, v. 15, n. 5, p. 267-271, 2007.

LAWTON, M. P.; BRODY, E. M. Assessment of older people: self-maintaining and instrumental activities of daily living. Gerontologist, v. 9, p. 179-186, 1969.

LIMA-COSTA, M. F.; BARRETO, S.; GIATTI, L.; UCHÔA, E. Socioeconomic circumstances and health among the brazilian elderly: a study using data from a National Household Survey. Cad Saude Publica, v. 19, n. 3, p. 745-757, 2003.

LINO, V. T. S.; PEREIRA, S. E. M.; CAMACHO, L. A. B.; RIBEIRO FILHO, S. T.; BUKSMAN, S. Cross-cultural adaptation of the independence in activities of daily living index (Katz Index). Cad Saude Publica, v. 24, n. 1, p. 103-112, 2008.

MARINHO, L. M.; VIEIRA, M. A.; COSTA, S. M.; ANDRADE, J. M. O. Degree of dependence of elderly residents in geriatric long-term care facilities in Montes Claros, MG. Rev Gaucha Enferm, v. 34, n. 1, p. 104-110, 2013.

MATSUDO, S. M. R. Avaliação do idoso: física e funcional. 3. ed. Londrina: Midiograf, 2010.

MELO, L. N. P.; SAINTRAIN, M. V. L. Epidemiological profile of elderly women assisted in the "Support Group for Prevention of Functional Disability". Rev Bras Promoç Saude, v. 22, n. 4, p. 251-258, 2009.

MILLÁN-CALENTI, J. C.; TUBÍO, J.; PITA-FERNÁNDEZ, S.; GONZÁLEZ-ABRALDES, I.; LORENZO, T.; FERNÁNDEZ-ARRUTY, T.; MASEDA, A. Prevalence of functional disability in activities of daily living (ADL), instrumental activities of daily living (IADL) and associated factors, as predictors of morbidity and mortality. Arch Gerontol Geriatr, v. 50, n. 3, p. 306-310, 2010.

MUIR, S. W.; GOPAUL, K.; ODASSO, M. M. M. The role of cognitive impairment in fall risk among older adults: a systematic review and meta-analysis. Age Ageing, v. 41, n. 3, p. 299-308, 2012.

OLIVEIRA, P. H.; MATTOS, I. E. Prevalence of functional disability and associated factors among institutionalized elders in the municipality of Cuiaba, state of Mato Grosso, Brazil, 20092010. Epidemiol Serv Saude, v. 21, n. 3, p. 395-406, 2012.

OLIVEIRA, D. L. C.; GORETTI, L. C.; PEREIRA, L. S. M. Performance in daily living activities and mobility among institutionalized elderly people with cognitive impairments: pilot study. Rev Bras Fisioter, v. 10, n. 1, p. 91-96, 2006.

PELEGRIN, A. K. A. P.; ARAÚJO, J. A.; COSTA, L. C.; CYRILlO, R. M. Z.; ROSSET, I.; Aged patients of a nursing home in Ribeirão Preto: levels of functional capacity. Arq Cienc Saude, v. 15, n. 4, p. 182-188, 2008. 
RAPOSO, P.; NOGUEIRA, D.; REIS, E.; SERRASQUEIRO, R. Nursing home residents: the dimension of frailty. Top Geriatr Rehabil, v. 33, n. 1, p. 72-82, 2017.

REBELATTO, J. R.; CALVO, J. I.; OREJUELA, J. R.; PORTILLO, J. C. Influence of a longterm physical activity program on hand muscle strength and body flexibility among elderly women. Rev Bras Fisioter, v. 10, n. 1, p. 127-132, 2006.

SUDRÉ, M. R. S.; REINERS, A. A. O.; NAKAGAWA, J. T. T.; AZEVEDO, R. S.; FLORIANO, L. A.; MORITA, L. H. M. Prevalence of dependency and associated risk factors in the elderly. Acta Paul Enferm, v. 25, n. 6, p. 947-953, 2012.

VERAS, R. P. Pais jovem com cabelos brancos: a saúde do idoso no Brasil. Rio de Janeiro: Relume Dumará, 1994. 